\title{
Retraction \\ Retracted: Clinical Significance of Resistin Expression in Osteoarthritis: A Meta-Analysis
}

\section{BioMed Research International}

Received 25 November 2020; Accepted 25 November 2020; Published 24 December 2020

Copyright (c) 2020 BioMed Research International. This is an open access article distributed under the Creative Commons Attribution License, which permits unrestricted use, distribution, and reproduction in any medium, provided the original work is properly cited.

BioMed Research Internationalhas retracted the article titled "Clinical Significance of Resistin Expression in Osteoarthritis: A Meta-Analysis" [1]. This article is one of a series of very similar meta-analyses written by different authors that were published in 2014 and 2015, characterized by the use of particular phrases [2]. The articles have the same structure, with the figures in the same order. The appearance of the figures and parts of the text are also similar.

The statistical tests in Figure 6 are underpowered, because they have less than 10 studies.

The original files submitted by the authors were edited by MedChina, a company previously alleged to be involved in the sale of articles [3]. The authors could not be contacted.

\section{References}

[1] X.-C. Li, F. Tian, and F. Wang, "Clinical significance of Resistin expression in osteoarthritis: a meta-analysis," BioMed Research International, vol. 2014, Article ID 208016, 11 pages, 2014.

[2] G. Filion, "A flurry of copycats on PubMed," The Grand Locus, 2014, http://blog.thegrandlocus.com/2014/10/a-flurry-ofcopycats-on-pubmed. View at: Google Scholar.

[3] C. Seife, "For Sale: "your name here" in a prestigious science journal," Scientific American, 2014, https://www.scientificamerican .com/article/for-sale-your-name-here-in-a-prestigious-sciencejournal/?redirect=1. View at: Google Scholar. 


\title{
Clinical Significance of Resistin Expression in Osteoarthritis: A Meta-Analysis
}

\author{
Xiao-Chuan Li, Feng Tian, and Fei Wang \\ Department of Hand \& Foot Surgery, Shengjing Hospital of China Medical University, Sanhao Street No. 36, Heping Distrct,
} Shenyang 110001, China

Correspondence should be addressed to Xiao-Chuan Li; lixiaochuang522@163.com

Received 19 July 2014; Accepted 2 September 2014; Published 29 October 2014

Academic Editor: Magali Cucchiarini

Copyright (C 2014 Xiao-Chuan Li et al. This is an open access article distributed under the Creative Commons Attribution License, which permits unrestricted use, distribution, and reproduction in any medium, provided the original work is properly cited.

Background. The objective of this study was to conduct a systematic review of literature evaluating human resistin expression as a diagnostic factor in osteoarthritis development and to quantify the overall diagnostic effect. Method. Relevant studies were identified and evaluated for quality through multiple search strategies. Studies analyzing resistin expression in the development of OA were eligible for inclusion. Data from eligible studies were extracted and included into the meta-analysis using a randomeffects model. Results. Four case-control studies consisting of a total of $375 \mathrm{OA}$ patients and 214 controls as well as three sex-stratified analyses composed of 53 males and 104 females were incorporated into our meta-analysis. Our results revealed that resistin levels were significantly higher in male OA subjects and OA patients overall. Country-stratified analysis yielded significantly different estimates in resistin levels between male OA subjects and female OA subjects in the Canadian subgroup but not among the French and USA subgroups. Based on the resistin levels in OA cases and controls, resistin levels were heightened in OA patients in the Dutch population. Conclusion. These results support the hypothesis that high expression of resistin represents a significant and reproducible marker of poor progression in OA patients, especially in males.

\section{Introduction}

Osteoarthritis (OA), characterized by articular cartilage loss, subchondral bone remodeling, soft tissue damage and inflammation, is the most common form of arthritis and a major cause of disability in the adult population $[1,2]$. OA is the main cause for hip and knee arthroplasties and therefore presents a major public health problem [3]. It is estimated by the World Health Organization Scientific Group on Rheumatic Diseases that $10 \%$ of the world's population over 60 have significant clinical problems that can be attributed to OA [4]. Data from Europe shows prevalence estimates ranging from $6 \%$ to $17 \%$ for knee $\mathrm{OA}$ and $2 \%$ to $10 \%$ for hip OA, indicating that OA has become the most frequent musculoskeletal disease [3]. Affecting 9.6\% of men and 18\% of women aged over 60 worldwide, symptomatic OA is one of the ten most disabling diseases with different proportions in each gender [5]. The etiology of osteoarthritis is still not fully understood because of its multifactorial effects, which can be considered the product of interactions between systemic and local factors within the joint [6,7]. Systemic risk factors include age, ethnicity, gender, and genetic variables, while local risk factors are obesity, previous knee injury, occupational activities, and so on [8]. As for genetic variables, various expressions of growth factors and cytokines have been observed in synovial fluid and synovial tissues from patients with $\mathrm{OA}$, within which resistin, an inflammatory cytokine, is one of those biomarkers [9-12].

Resistin, a kind of cysteine-rich secretory protein belonging to the resistin-like molecule found in the inflammatory zone (RELM/FIZZ) family, consists of 108 amino acid prepeptides which circulate as a dimeric protein consisting of two 92-amino acid polypeptides in human blood [13, 14]. Unlike rodents, whose main source of resistin is adipocytes, the main source of resistin in humans is mononuclear cells within and outside adipose tissue [15]. It has been reported that resistin takes part in glucose and peripheral lipid metabolism, which are related to metabolic diseases, diabetes, and hyperlipidemia [16]. Primarily produced by macrophages, there is some evidence that resistin may be involved with 
other immune cells and secrete proinflammatory factors in inflammatory diseases [17]. Studies have demonstrated that resistin is an inflammatory mediator and a biomarker of cardiovascular diseases [18]. Upregulation of resistin is in inverse association with most alimentary tract disorders in animal and human studies [13]. Resistin also plays important roles in nonalcoholic fatty liver disease, autoimmune disease, malignancy, asthma, chronic kidney disease, and bone metabolism [19, 20]. Evidence has shown that higher serum levels of resistin in patients with severe osteoarthritis (OA) compared to controls with no $\mathrm{OA}$ and resistin are detected in both serum and synovial fluid, proving its systematical and local involvement in inflammatory changes of OA $[12,21]$. This could be explained by the role of resistin as an inducer of proinflammatory cytokines including TNF- $\alpha$, IL-6, and IL12 through the nuclear factor $-\kappa \mathrm{B}$ signal pathway in diverse inflammatory conditions [22]. Another prevailing hypothesis is that obesity, which has a close link with resistin, may cause increased loads on joint surfaces leading to cartilage wear, a risk for OA, suggesting that resistin may be related with $\mathrm{OA}$ through its metabolism functions [23]. Nevertheless, there exist some contrary conclusions and inconsistent studies about these discoveries [21, 24]. To perform a systematical meta-analysis to evaluate the connection between resistin expression and pathogenesis of $\mathrm{OA}$ is of great significance.

\section{Materials and Methods}

This meta-analysis was conducted according to the guidelines of the preferred reporting items for systematic reviews and meta-analyses (PRISMA) statement on the quality of published systematic reviews and meta-analyses [25].

2.1. Search Strategy. Potentially relevant studies were identified by a comprehensive search of the literature without language or country restrictions covering the following computerized bibliographic databases: MEDLINE (1966 2014), Science Citation Index (1945 2014), the Cochrane Library (Oxford, UK, Issue 12, 2014), PubMed (1966 2014), Embase (1974 2014), CINAHL (1982 2014), and the Current Contents Index (1995 2014). Three Chinese databases (Chinese Biomedical, 1978 2014; the Chinese Journal FullText, 1980 2014; and the Weipu Journal, 1989 2014) were also applied to identify Chinese-language articles. We used the following medical subject headings and free language terms in conjunction with a highly sensitive search strategy: "stomach neoplasms" or "Osteoarthritis, Knee" or "Osteoarthritis, Hip" or "Osteoarthritis" or "knee osteoarthritis" or "spine osteoarthritis" or "hip osteoarthritis" or "spinal osteoarthritis" or "lumbar osteoarthritis" or "coxarthrosis" and "Resistin." Additionally, reference lists of the relevant studies were selected from the electronic debates and searched manually to find additional work.

2.2. Inclusion and Exclusion Criteria. To be included in the systematic review, retrieved studies had to be assessed for their suitability by meeting the following criteria: (1) the study was conducted within a human population and published in a peer-reviewed journal; (2) only case-control or cohort studies examining the association of resistin level in the pathogenesis in OA were incorporated into the metaanalysis; (3) all patients were confirmed to have OA in accordance with the definitions and classification provided by the Diagnostic and Therapeutic Criteria Committee of the American Rheumatism Association [26]; (4) the article must present original data and supply sufficient information on resistin levels; (5) if studies provided overlapping data, we would choose the study that had the largest number. The major exclusion criteria in this systematic review were (1) the article that did not satisfy the current inclusion criteria; (2) some publications presenting nonoriginal data, such as letters, editorials, abstracts, reviews, meta-analysis opinion papers, or proceedings; (3) unpublished sources of data; (4) duplicated publications or studies without extractable, numerical data; and (5) subgroup analyses of the included trials. With the help of these inclusion criteria, the title and abstract of all the articles were evaluated based on relevance. From the selected articles, the full texts were reviewed to decide their eligibility for inclusion.

2.3. Study Quality and Data Extraction. Two experienced reviewers independently assessed the methodological quality of the included trials using the critical appraisal skills program (CASP, Milton Keynes Primary Care Trust, 2002, Institute of Health Sciences, Oxford) to ensure consistency in reviewing and reporting results (available at http://www.phru.nhs.uk/casp/qualitat.htm). Each paper was assigned a level of evidence as follows: (1) at least one good systematic review; (2) at least one good randomized controlled trial; (3) well-designed interventional studies without randomization; (4) well-designed observational studies; (5) influential reports or expert opinion.

Two reviewers assessed the studies independently based on the inclusion/exclusion criteria mentioned before in the methods section. We used a standardized data form to collect the following descriptive information: surname and initials of the first author, the year of publication or submission, journal, source country, racial descent of study population, language of publication, study design, number of subjects, demographic variables of the subjects, sample used to detect the resistin levels, detection methods of resistin levels, resistin levels, confirmation of diagnosis, and so forth. Disagreement on the inclusion of a single study was settled by discussion or a third investigator was consulted.

2.4. Statistical Analysis. The effect size, represented by a standardized mean difference (SMD) for resistin levels, was calculated. A 95\% confidence interval (95\% CI) was calculated for the summary SMD by the use of the $Z$ test. A test for heterogeneity between trials included for each comparison was performed by the use of Cochran's Qstatistic and $I^{2}$ tests [27]. If the Q-test showed evidence of $P<0.05$ or the $I^{2}$ test exhibited $>50 \%$, indicating maximal heterogeneity among the included studies, we performed a metaregression analysis with a random-effects model to explore the sources of heterogeneity. Otherwise, SMDs were 


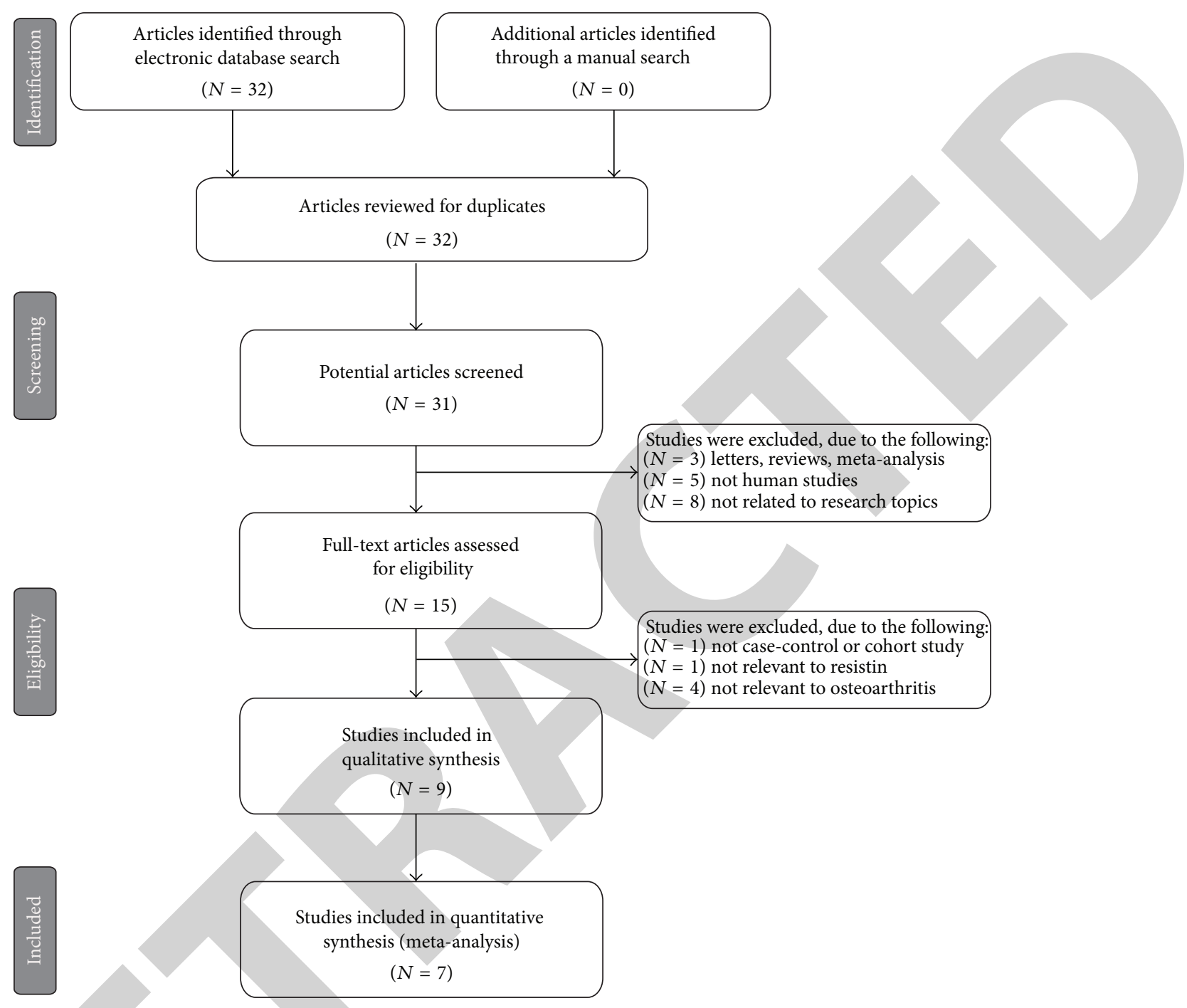

FIGURE 1: Flow chart showing study selection procedure. Seven clinical studies were included in this meta-analysis.

pooled in accordance with the fixed-effects model [28, 29]. When significant heterogeneity existed, the differences in resistin levels (and 95\% CI) were assessed for using subgroups with different explanatory variables. Additionally, sensitivity analysis was conducted to investigate the stability condition of our present results. By using a stepwise process, every study was individually omitted for the purposes of reflecting the effect of each individual paper and dataset on the pooled overall OR in our meta-analysis. Further, Egger's linear regression test with visual inspection of the funnel plot was applied to detect potential publication bias [30, 31]. Statistical analyses were conducted with the STATA statistical software (Version 12.0, Stata Corporation, College Station, TX, USA).

\section{Results}

3.1. Description of Included Studies. The combined electronic and manual search initially resulted in 32 potentially eligible articles. One duplicate study was removed, and then the retrieved studies were screened by title and abstract for relevance. 16 irrelevant articles were excluded. Then we systematically reviewed the remaining 15 articles with a fulltext reading. Subsequently, 6 articles were deemed unsuitable and were therefore excluded; thus 9 articles were identified to be included in qualitative analysis. In addition, another 2 studies were excluded due to lack of data integrity after a more careful assessment of the remaining articles. Finally, 7 casecontrol studies consisting of $375 \mathrm{OA}$ patients and 214 controls, including 3 sex-stratified analyses composed of 53 males and 104 females, were incorporated into the current meta-analysis with sample sizes ranging from 34 to 304 subjects [12, 21$24,32,33]$. A flow diagram of the study selection's progression and the main reasons for exclusion is displayed in Figure 1. All eligible studies were published from 2008 to 2014 (Figure 2). Most enrolled papers showed moderate to high quality.

Of the 7 studies, all 3 sex-stratified studies were performed in Caucasian populations (Canada (Perruccio), USA (Massengale), and France (Gegout)). Blood samples were used to detection resistin levels by enzyme-linked 


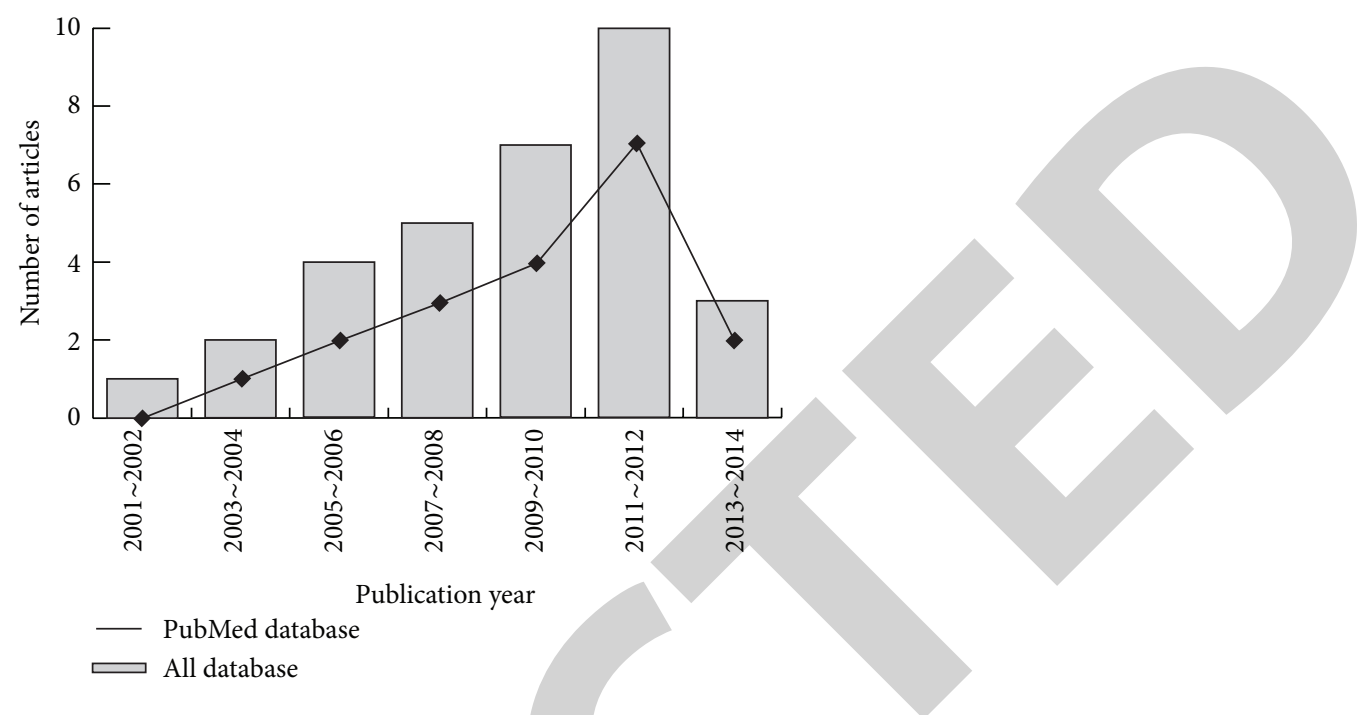

FIgURE 2: The distribution of topic-related literature in the electronic databases over the last decade.

immunosorbent assay (ELISA). Meanwhile, with respect to the 4 case-control studies, 2 studies were conducted in Caucasian populations (The Netherlands (de Boer) and Czech (Filkova)), while one was conducted among Asians (Korea (Choe)), and the final study was performed in a mixed ethnicity population (Beekhuizen). For the demographic variables, Choe et al. failed to obtain the gender information in both cases and controls while Filkova et al. lost the age information in both cases and controls. In addition, synovial fluid and blood samples were used to detect the resistin level with ELISA. Tables 1 and 2 showed the baseline characteristics and resistin levels of the individual studies.

3.2. Quantitative Data Synthesis. In the meta-analysis, the following analyses were performed with a random-effects model for the evidence of Q-test and $I^{2}$ test (male versus female: $I^{2}=93.6 \%, P<0.001$; cases versus controls: $I^{2}=96.9 \%, P<0.001$, resp.). Additionally, based on the significant heterogeneity existing across the different populations observed in these studies, these groups of studies were stratified by country (male versus female: France, USA, and Canada subgroups; case versus controls: The Netherlands, Korea, and Czech subgroups, resp.). When concerned about the correlation between resistin levels and male/female OA subjects, results of the present study revealed that resistin showed significantly higher levels in male OA subjects in contrast to female OA subjects, according to the pooled SMDs in the three included studies (SMD $=0.45,95 \% \mathrm{CI}=0.08 \sim$ $0.83, P=0.018)$. In addition, with respect to the difference in resistin levels between $\mathrm{OA}$ cases and the controls, findings in the current meta-analysis demonstrated that OA patients had considerably higher resistin levels than those in the controls; specifically, the pooled SMD of resistin levels between OA cases and the controls was $0.60(95 \% \mathrm{CI}=0.43 \sim 0.77)(P<$ 0.001) (Figure 3).

In the country-stratified subgroups, the results only yielded significantly different estimates in resistin levels between male OA subjects and female OA subjects in the Canadian subgroup $(\mathrm{SMD}=2.95,95 \% \mathrm{CI}=1.97 \sim 3.93, P<$ 0.001 ), but a similar correlation was not observed in the French subgroup and the USA subgroup (all $P>0.05$ ). Based on the resistin levels in OA cases and controls, we found that resistin levels were heightened in OA patients in comparison with the controls in the Dutch population (SMD $=0.32,95 \%$ $\mathrm{CI}=0.10 \sim 0.54, P=0.004)$ and the Korean population (SMD $=2.20,95 \% \mathrm{CI}=1.80 \sim 2.59, P<0.001)$. This difference did not manifest in the Czech population $(P>0.05)$ (Figure 4$)$.

We conducted further sensitivity analyses to determine whether review conclusions were affected by the inclusion of a single study. Our findings suggested that no single study had an effect on the pooled SMDs in the current metaanalysis (Figure 5). Finally, Egger's regression test showed no evidence of asymmetrical distribution in the funnel plot in the association of resistin levels and the pathogenesis of OA (male versus female: $t=1.46, P=0.382$; cases versus controls: $t=1.20, P=0.295$ ) (Figure 6).

\section{Discussion}

We conducted the present meta-analysis in an attempt to determine the connection between resistin expression and the pathogenesis of OA. The major findings of our metaanalysis indicated that resistin expression in patients with OA was significantly higher than those of healthy controls, implying that resistin expression may be closely related to the development of OA. Although accumulated evidence has demonstrated that various immunological and inflammatory mechanisms may be conductive towards the development and progression of OA, very little is known about the exact role of resistin in the pathogenesis of OA. Resistin is widely accepted as a member of the found in the FIZZ protein family and is generally secreted by human peripheral blood mononuclear cells [34]. In addition, resistin is also a dimeric protein generated by adipocytes and macrophages, capable of 


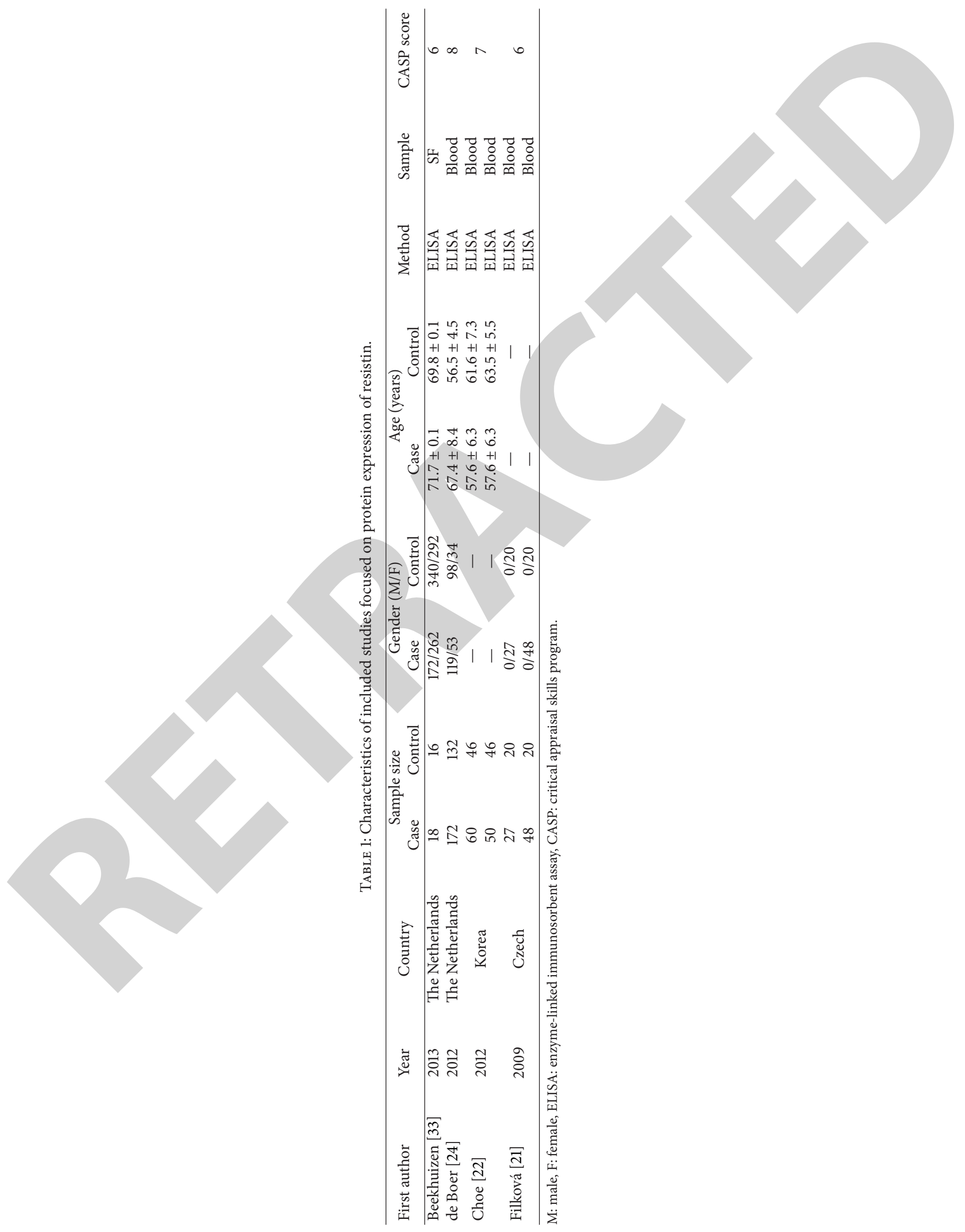




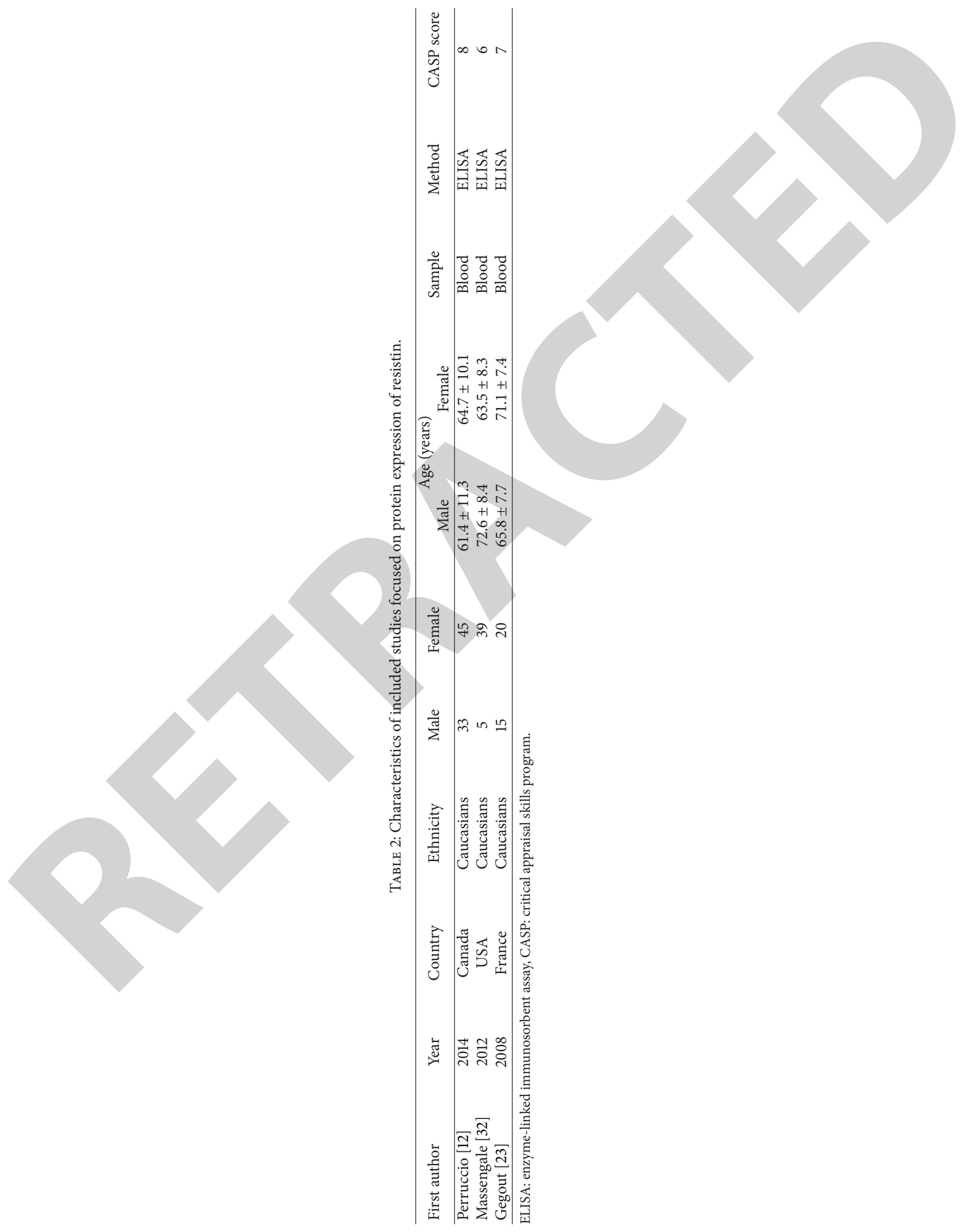


Resistin

(male versus female)

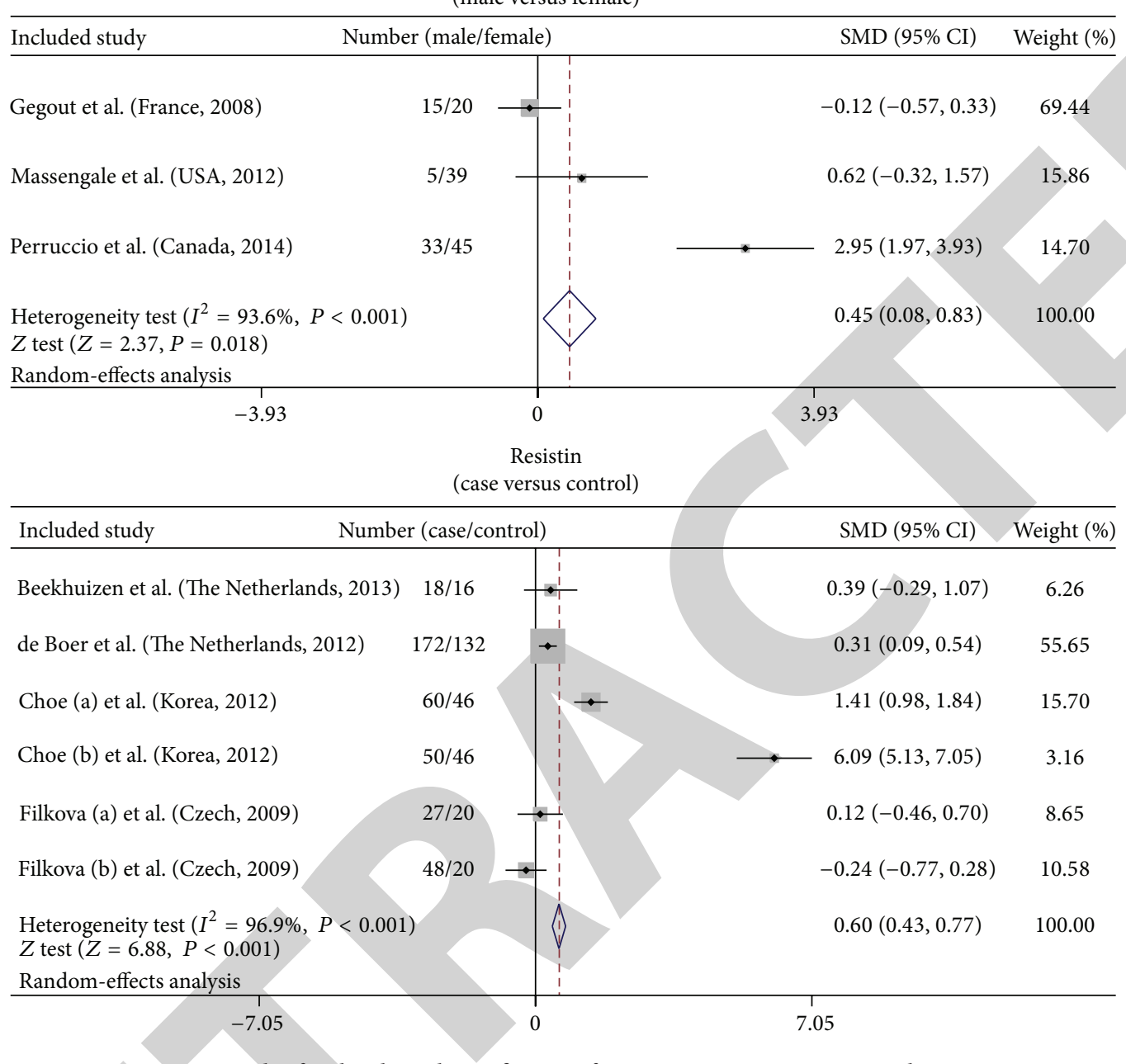

FIGURE 3: Forest plot for the clinical significance of resistin expression in osteoarthritis patients.

inducing insulin resistance in mice [35]. In human beings, resistin seems to play a more crucial role in inflammatory processes than it does in insulin resistance since serum resistin levels are linked more to subclinical inflammation than to insulin resistance [36]. Previous studies have reported that healthy mice injected in their knee joints with resistin exhibited an arthritis-like condition with leukocyte infiltration of synovial tissues, hypertrophy of the synovial layer, and pannus formation $[37,38]$. More importantly, resistin is suggested to participate in inflammatory changes of OA systemically and locally due to the fact that resistin is observed in both serum and synovial fluid and its expression level is increased significantly after traumatic injury $[22,39]$. In this regard, it is possible to hypothesize that resistin expression may be strongly connected with the pathogenesis of OA. In accordance with our findings, Choe et al. also found that resistin may be strongly associated with radiographic changes in hand $\mathrm{OA}$, demonstrating that resistin expression may have an important role in predicting radiographic outcomes in patient with hand OA [22]. Gegout et al. also found that resistin may play a central role in initiating and promoting the development of OA and may be one of the major connections between metabolic disorders related with obesity and the destruction of joint cartilage [23]. Furthermore, our results illustrated that plasma resistin levels of male patients with $\mathrm{OA}$ were increased significantly compared with those of female patients, supporting the notion that males may be more prone to develop OA than females. In a previous study carried out by Massengale et al., the data indicated that the mean resistin of male patients with $\mathrm{OA}$ was $9.4 \mathrm{ng} / \mathrm{mL}$, while that of female patients was only $7.3 \mathrm{ng} / \mathrm{mL}$ [32].

We comprehensively implemented subgroup analysis in order to further elucidate the potential relationship between resistin expression and the development of OA. The results of subgroup analysis stratified by country revealed that OA patients exhibited higher resistin expression in both Dutch and Korean populations. In addition, our findings showed that the resistin levels of male patients with OA were apparently higher than those of female patients in the Canadian population. In short, our discovery conformed to previous findings showing that resistin expression may be closely implicated in the pathogenesis of $\mathrm{OA}$, suggesting that 
Resistin

(country: male versus female)

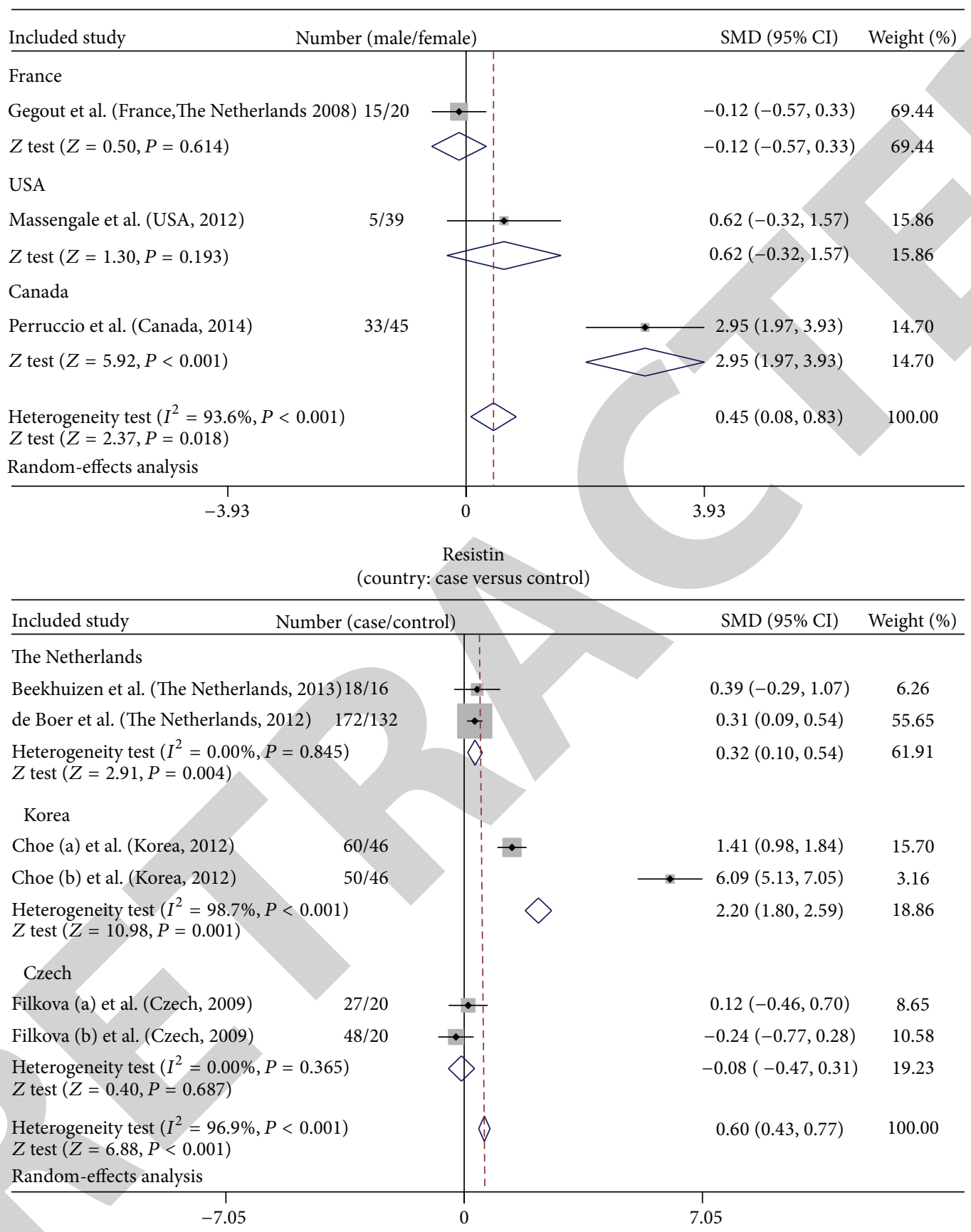

FIGURE 4: Subgroup analyses for the clinical significance of resistin expression in osteoarthritis patients.

the detection of expression levels of resistin may be valuable in the early prediction of disease severity and prognoses of OA patients for the purpose of avoiding poor clinical outcomes.

Similarly, there did exist some limitations in the current meta-analysis that should be taken into consideration. First, even though we attempted to utilize the PubMed, EMBASE, and several other Chinese databases as completely as possible by performing our literature search, we may not have been able to minimize publication bias. Several unpublished papers and abstracts were not taken into account because the data required for the inclusion and exclusion criteria was unavailable. A second potential source of bias may be related to the method used to extrapolate the OR due to the existence of potential heterogeneity. Additionally, only studies that found significant results in univariate analysis performed multivariate analysis, though each study already adjusted for different covariates, and thus the pooled results may produce 


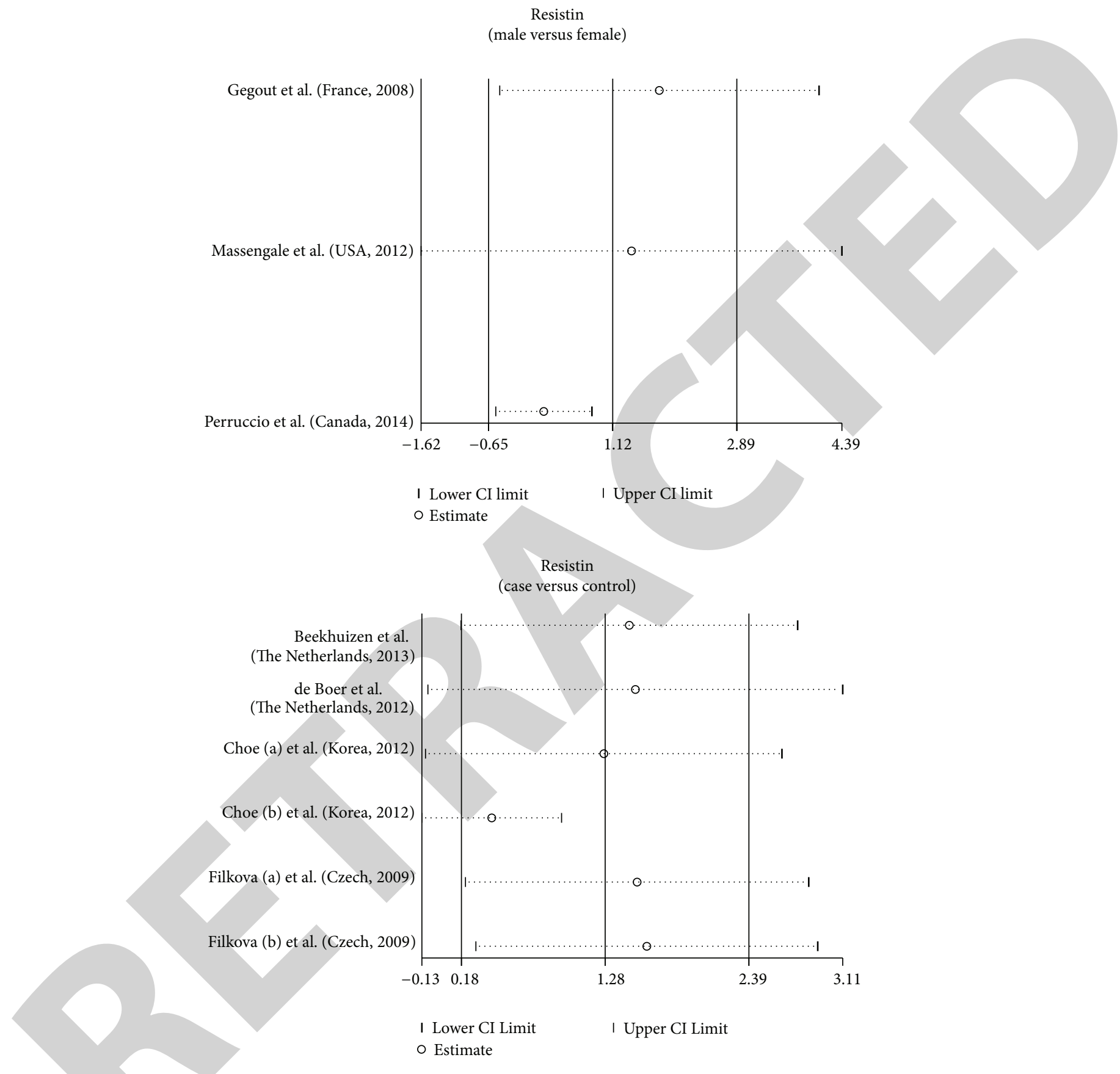

FIGURE 5: Sensitivity analysis of the summary odds ratio coefficients for the clinical significance of resistin expression in osteoarthritis patients. Meta-analysis random-effects estimates (exponential form) were used. The two ends of the dotted lines represent the $95 \%$ CI.

biases. Thirdly, although our search of eligible literature was conducted without language restrictions, only studies written in Chinese and English were included in the meta-analysis. Nevertheless, publication bias was not discovered when using Egger's test, indicating that the statistical data obtained from those included literatures may approximate the actual results. Despite the above limitations, this is the first example of meta-analysis on the association of resistin expression with the development of OA. Most importantly, our meta-analysis also displayed significant advantages. With the application of strict inclusion and exclusion criteria in our meta-analysis, the quality of studies included was satisfactory and strictly met those criteria. In addition, we also performed subgroup analysis by measuring extrinsic factors that may have an effect on the expression of resistin.

In conclusion, our meta-analysis indicated that high resistin expression was a strong predictor of all two comparison outcomes. The critical role of resistin in OA diagnosis may contribute to its clinical utility. Our conclusions, however, need to be confirmed considering the limitations 


\section{Resistin \\ (male versus female)}

(Egger's test: $t=1.46, P=0.382$ )

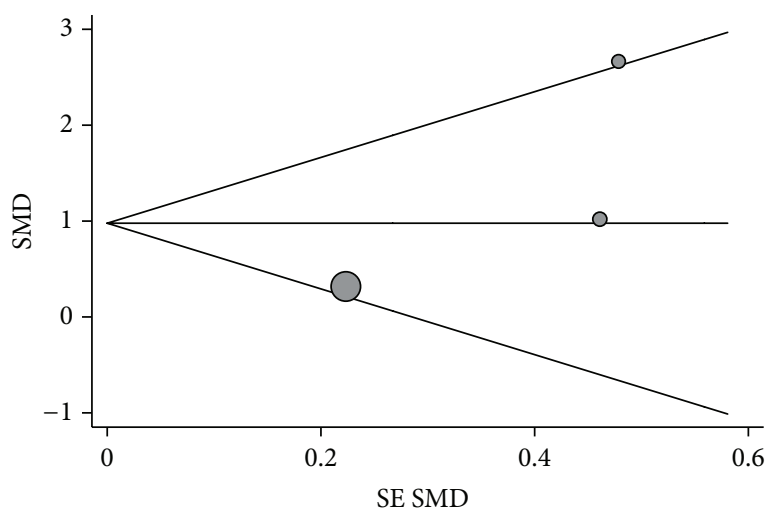

Resistin
(case versus control)

(Egger's test: $t=1.20, P=0.295$ )

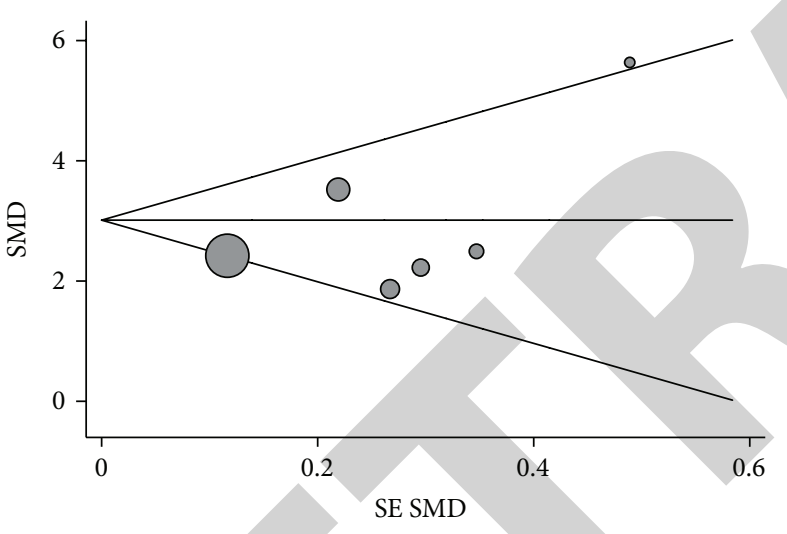

FIGURE 6: Funnel plots for the clinical significance of resistin expression in osteoarthritis patients. Each point represents a separate study for the indicated association. Horizontal line, mean magnitude of the effect.

of the present analysis. Further research with standardized, unbiased methods and larger sample sizes are required for deeper analysis.

\section{Conflict of Interests}

The authors have declared that no competing interests exist.

\section{Acknowledgment}

The authors would like to acknowledge the reviewers for their helpful comments on this paper.

\section{References}

[1] C. A. Karvonen-Gutierrez, S. D. Harlow, P. Mancuso, J. Jacobson, C. F. M. De Leon, and B. Nan, "Association of leptin levels with radiographic knee osteoarthritis among a cohort of midlife women," Arthritis Care and Research, vol. 65, no. 6, pp. 936-944, 2013.

[2] M. B. Goldring and S. R. Goldring, "Articular cartilage and subchondral bone in the pathogenesis of osteoarthritis," Annals of the New York Academy of Sciences, vol. 1192, pp. 230-237, 2010.

[3] F. Guillemin, A. C. Rat, B. Mazieres et al., "Prevalence of symptomatic hip and knee osteoarthritis: a two-phase populationbased survey," Osteoarthritis and Cartilage, vol. 19, no. 11, pp. 1314-1322, 2011

[4] D. Pereira, B. Peleteiro, J. Araújo, J. Branco, R. A. Santos, and E. Ramos, "The effect of osteoarthritis definition on prevalence and incidence estimates: a systematic review," Osteoarthritis and Cartilage, vol. 19, no. 11, pp. 1270-1285, 2011.

[5] S. A. Hardcastle, C. L. Gregson, K. C. Deere, G. D. Smith, P. Dieppe, and J. H. Tobias, "High bone mass is associated with an increased prevalence of joint replacement: a case-control study," Rheumatology, vol. 52, no. 6, pp. 1042-1051, 2013.

[6] A. Heijink, A. H. Gomoll, H. Madry et al., "Biomechanical considerations in the pathogenesis of osteoarthritis of the knee," Knee Surgery, Sports Traumatology, Arthroscopy, vol. 20, no. 3, pp. 423-435, 2012.

[7] A. Mobasheri, "Osteoarthritis year 2012 in review: biomarkers," Osteoarthritis and Cartilage, vol. 20, no. 12, pp. 1451-1464, 2012.

[8] R. K. Chaganti and N. E. Lane, "Risk factors for incident osteoarthritis of the hip and knee," Current Reviews in Musculoskeletal Medicine, vol. 4, no. 3, pp. 99-104, 2011.

[9] J. H. Ku, C. K. Lee, B. S. Joo et al., "Correlation of synovial fluid leptin concentrations with the severity of osteoarthritis," Clinical Rheumatology, vol. 28, no. 12, pp. 1431-1435, 2009.

[10] A. Koskinen, K. Vuolteenaho, T. Moilanen, and E. Moilanen, "Resistin as a factor in osteoarthritis: synovial fluid resistin concentrations correlate positively with interleukin 6 and matrix metalloproteinases MMP-1 and MMP-3," Scandinavian Journal of Rheumatology, vol. 43, no. 3, pp. 249-253, 2014.

[11] S. M. H. Fadda, S. M. Gamal, N. Y. Elsaid, and A. M. Mohy, "Resistin in inflammatory and degenerative rheumatologic diseases: relationship between resistin and rheumatoid arthritis disease progression," Zeitschrift für Rheumatologie, vol. 72, no. 6, pp. 594-600, 2013.

[12] A. V. Perruccio, N. N. Mahomed, V. Chandran, and R. Gandhi, "Plasma adipokine levels and their association with overall burden of painful joints among individuals with hip and knee osteoarthritis," Journal of Rheumatology, vol. 41, no. 2, pp. 334337, 2014.

[13] E. K. Tiaka, A. C. Manolakis, A. N. Kapsoritakis, and S. P. Potamianos, "The implication of adiponectin and resistin in gastrointestinal diseases," Cytokine and Growth Factor Reviews, vol. 22, no. 2, pp. 109-119, 2011.

[14] A. Sood and S. A. Shore, "Adiponectin, leptin, and resistin in asthma: basic mechanisms through population studies," Journal of Allergy, vol. 2013, Article ID 785835, 15 pages, 2013.

[15] M. Filková, L. Senolt, and J. Vencovský, “The role of resistin in inflammatory myopathies," Current Rheumatology Reports, vol. 15, no. 6, p. 336, 2013.

[16] R. Nogueiras, M. G. Novelle, M. J. Vazquez, M. Lopez, and C. Dieguez, "Resistin: regulation of food intake, glucose homeostasis and lipid metabolism," Endocrine Development, vol. 17, pp. 175-184, 2009.

[17] R. Krysiak, G. Handzlik-Orlik, and B. Okopien, "The role of adipokines in connective tissue diseases," European Journal of Nutrition, vol. 51, no. 5, pp. 513-528, 2012. 
[18] S. E. Lee and H.-S. Kim, "Human resistin in cardiovascular disease," Journal of Smooth Muscle Research, vol. 48, no. 1, pp. 27-35, 2012.

[19] M. S. Jamaluddin, S. M. Weakley, Q. Yao, and C. Chen, "Resistin: functional roles and therapeutic considerations for cardiovascular disease," British Journal of Pharmacology, vol. 165, no. 3, pp. 622-632, 2012.

[20] M. Filkova, M. Haluzik, S. Gay, and L. Senolt, "The role of resistin as a regulator of inflammation: Implications for various human pathologies," Clinical Immunology, vol. 133, pp. 157-170, 2009.

[21] M. Filková, M. Lišková, H. Hulejová et al., "Increased serum adiponectin levels in female patients with erosive compared with non-erosive osteoarthritis," Annals of the Rheumatic Diseases, vol. 68, no. 2, pp. 295-296, 2009.

[22] J.-Y. Choe, J. Bae, H.-Y. Jung, S.-H. Park, H.-J. Lee, and S.-K. Kim, "Serum resistin level is associated with radiographic changes in hand osteoarthritis: cross-sectional study," Joint Bone Spine, vol. 79, no. 2, pp. 160-165, 2012.

[23] P. P. Gegout, P.-J. Francin, D. Mainard, and N. Presle, "Adipokines in osteoarthritis: friends or foes of cartilage homeostasis?" Joint Bone Spine, vol. 75, no. 6, pp. 669-671, 2008.

[24] T. N. de Boer, W. E. van Spil, A. M. Huisman et al., "Serum adipokines in osteoarthritis; comparison with controls and relationship with local parameters of synovial inflammation and cartilage damage," Osteoarthritis and Cartilage, vol. 20, no. 8, pp. 846-853, 2012.

[25] N. Panic, E. Leoncini, G. De Belvis, W. Ricciardi, and S. Boccia, "Evaluation of the endorsement of the preferred reporting items for systematic reviews and meta-analysis (PRISMA) statement on the quality of published systematic review and meta-analyses," PLoS ONE, vol. 8, no. 12, Article ID e83138, 2013.

[26] R. Altman, E. Asch, and D. Bloch, "Development of criteria for the classification and reporting of osteoarthritis. Classification of osteoarthritis of the knee," Arthritis and Rheumatism, vol. 29, no. 8, pp. 1039-1049, 1986.

[27] E. Zintzaras and J. P. A. Ioannidis, "HEGESMA: genome search meta-analysis and heterogeneity testing," Bioinformatics, vol. 21, no. 18, pp. 3672-3673, 2005.

[28] E. Zintzaras and J. P. A. Ioannidis, "Heterogeneity testing in meta-analysis of genome searches," Genetic Epidemiology, vol. 28, no. 2, pp. 123-137, 2005.

[29] J. P. T. Higgins and S. G. Thompson, "Quantifying heterogeneity in a meta-analysis," Statistics in Medicine, vol. 21, no. 11, pp. 15391558, 2002.

[30] F. Song and S. Gilbody, "Bias in meta-analysis detected by a simple, graphical test. Increase in studies of publication bias coincided with increasing use of meta-analysis," British Medical Journal, vol. 316, no. 7129, p. 471, 1998.

[31] J. L. Peters, A. J. Sutton, D. R. Jones, K. R. Abrams, and L. Rushton, "Comparison of two methods to detect publication bias in meta-analysis," Journal of the American Medical Association, vol. 295, no. 6, pp. 676-680, 2006.

[32] M. Massengale, B. Lu, J. J. Pan, J. N. Katz, and D. H. Solomon, "Adipokine hormones and hand osteoarthritis: radiographic severity and pain," PLoS ONE, vol. 7, no. 10, Article ID e47860, 2012.

[33] M. Beekhuizen, L. M. Gierman, W. E. van Spil et al., "An explorative study comparing levels of soluble mediators in control and osteoarthritic synovial fluid," Osteoarthritis and Cartilage, vol. 21, no. 7, pp. 918-922, 2013.
[34] L. Patel, A. C. Buckels, I. J. Kinghorn et al., "Resistin is expressed in human macrophages and directly regulated by PPAR $\gamma$ activators," Biochemical and Biophysical Research Communications, vol. 300, no. 2, pp. 472-476, 2003.

[35] C. M. Steppan and M. A. Lazar, "Resistin and obesity-associated insulin resistance," Trends in Endocrinology and Metabolism, vol. 13, no. 1, pp. 18-23, 2002.

[36] L. Šenolt, D. Housa, Z. Vernerová et al., "Resistin in rheumatoid arthritis synovial tissue, synovial fluid and serum," Annals of the Rheumatic Diseases, vol. 66, no. 4, pp. 458-463, 2007.

[37] F. Lago, C. Dieguez, J. Gómez-Reino, and O. Gualillo, “Adipokines as emerging mediators of immune response and inflammation," Nature Clinical Practice Rheumatology, vol. 3, no. 12, pp. 716-724, 2007.

[38] O. Gabay, D. J. Hall, F. Berenbaum, Y. Henrotin, and C. Sanchez, "Osteoarthritis and obesity: experimental models," Joint Bone Spine, vol. 75, no. 6, pp. 675-679, 2008.

[39] J. H. Lee, T. Ort, K. Ma et al., "Resistin is elevated following traumatic joint injury and causes matrix degradation and release of inflammatory cytokines from articular cartilage in vitro," Osteoarthritis and Cartilage, vol. 17, no. 5, pp. 613-620, 2009.

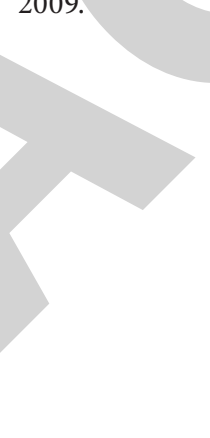

